\title{
Studies on the Role of Angiotensin in Experimental Renovascular Hypertension: an Immunologic Approach
}

\author{
A. R. Christlieb, T. U. L. Biber, and R. B. Hrckier \\ From the Hypertension Unit and the Department of Medicine, Peter Bent \\ Brigham Hospital, and Harvard Medical School, \\ Boston, Massachusetts 02115
}

A B S T RACT The role of angiotensin in three forms of experimental hypertension was assessed in rats. First, the acute blood pressure response to injected angiotensin amide and angiotensin acid was determined. Rats made hypertensive with deoxycorticosterone and saline showed exaggerated responses; rats made hypertensive by clipping one renal artery showed depressed responses; and rats made hypertensive by clipping one renal artery and contralateral nephrectomy showed normal responsivity to angiotensin amide but depressed responsivity to angiotensin acid. These findings suggested that different mechanisms may be involved in the three types of hypertension studied.

To assess the role of angiotensin in these hypertensive rats the blood pressure response, the presence of antibodies determined by radioimmune techniques, and the degree of refractoriness to injected angiotensin after immunization with angiotensin were studied. None of six rats made hypertensive by deoxycorticosterone and saline, and none of five mock immunized rats with renal hypertension of both types had a fall in blood pressure. By contrast, of the 20 rats with both types of renal hypertension in which antibody determinations were made, 11 had developed a significant antibody titer, of which seven showed a significant reduction in blood pressure at the time of antibody determination, and three of the remaining four had a significant blood pressure reduction earlier in their course. None of the nine renal hypertensive rats without demonstrable antibodies had a reduced blood pressure at the time of antibody determination, and only one had an earlier reduction in

This work was presented in part at the 60th Annual Meeting of the American Society for Clinical Investigation, Atlantic City, May 1968 and abstracted in $1968 \mathrm{~J}$. Clin. Invest. 49: 19a, and in 1967 Clin. Res. 15: 198.

Dr. R. B. Hickler is an Investigator of the Howard Hughes Medical Institute.

Received for publication 31 December 1968 and in revised form 28 March 1969. blood pressure. The renal hypertensive rats were all refractory to injected angiotensin after immunization.

These results suggest a primary role for angiotensin in both forms of renal hypertension.

\section{INTRODUCTION}

Evidence for an association between the renin-angiotensin system and the hypertension seen after constriction of a renal artery has been accumulating since 1898 (1-7). The most direct evidence for this association has been the production of antirenin, which results in the neutralization of the pressor effect of injected renin in the assay dog and which is associated with a lowering of the arterial pressure in renal hypertensive dogs, rats, and monkeys (8-13). However, nonspecificity of this response for renal hypertension is suggested by studies showing a reduction in the blood pressure of spontaneously hypertensive and pyelonephritic hypertensive dogs with the production of antirenin (14).

The production of hypertension and hypertensive vascular disease after injections of homologous crude renin into uninephrectomized rats provides further evidence that the renin-angiotensin system may be instrumental in producing renal hypertension (15-16). Recently, the blood pressure of rats with acute and chronic renal hypertension has been reduced by a renin-inhibiting phospholipid isolated from kidneys (17).

Although angiotensin has been thought to be the ultimate mediator of renovascular hypertension, definite evidence for its role in this disease has not been established. With the advent of conjugates of angiotensin capable of producing angiotensin antibodies (18-23) which are biologically effective (24), an immunologic approach to the role of angiotensin in the pathogenesis of renovascular hypertension seemed feasible.

This work is divided into two parts. Part I assesses endogenous angiotensin production in experimentally 


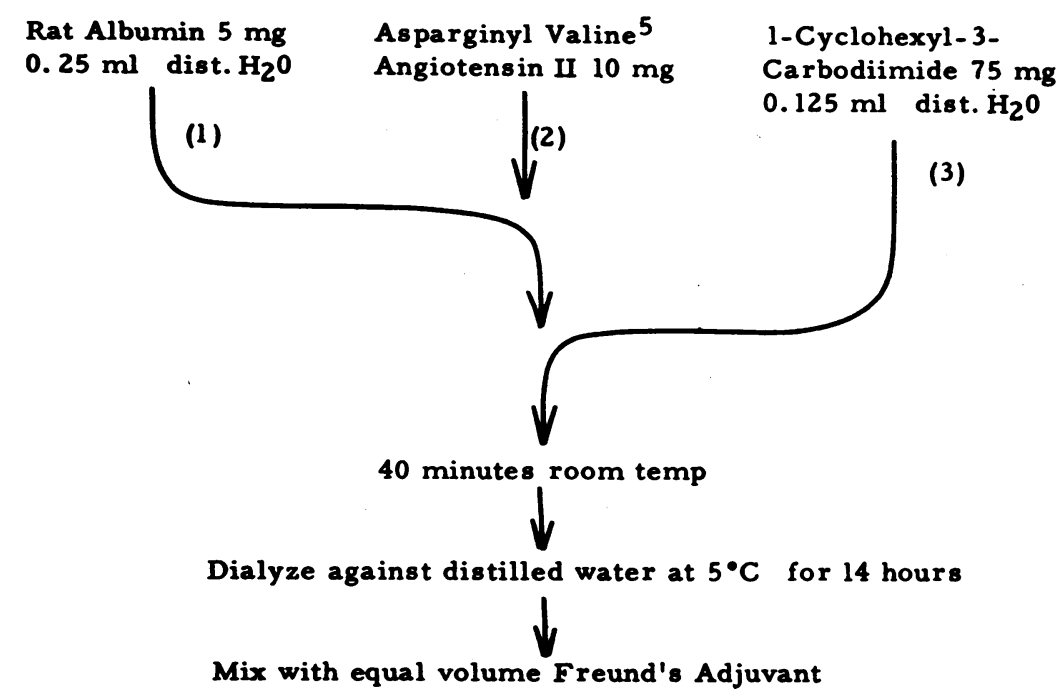

FIGURE 1 Preparation of the angiotensin antigen.

hypertensive rats indirectly by recording the blood pressure sensitivity to injected angiotensin, and Part II assesses the acute and chronic blood pressure response to active immunization against angiotensin in these rats.

\section{METHODS}

Part I. Four groups of male albino Sprague-Dawley rats weighing $250-350 \mathrm{~g}$ were studied. Group I consisted of eight normal rats; Group II of seven rats made hypertensive by unilateral nephrectomy, deoxycorticosterone acetate (DOCA) $5 \mathrm{mg}$ intramuscularly three times/wk, and fluid intake as $0.8 \%$ saline throughout the period of study (DOCA rats); Group III of seven rats made hypertensive by placing a constricting silver clip on the left renal artery together with contralateral nephrectomy (GN rats); and Group IV of 12 rats made hypertensive by placing a constricting silver clip on the left renal artery ( $G$ rats). After blood pressures of $170-260$ were recorded in Groups II, III, and IV by the indirect caudal method (Caudal Plethysmograph System, Model 320-1, Decker Corp., BalaCynwyd, $\mathrm{Pa}$.), each rat was anesthetized with sodium pentobarbital $30 \mathrm{mg} / \mathrm{kg}$ intraperitoneally which lowered the mean blood pressure to $40-120 \mathrm{~mm} \mathrm{Hg}$. The right carotid artery was canulated with a polyethylene catheter for direct recording of the blood pressure on a Bird Kymograph (Catalogue \#70-060, Phipps \& Bird, Richmond, Va.) fitted with a Palmer mercury manometer (Palmer Instruments, Inc., Cincinnati, Ohio). The left jugular vein was similarly catheterized for pharmacologic injections using Micro SyringeBurettes (California Laboratory Equipment Co., Berkeley, Calif.) capable of delivering accurately as little as 0.0001 $\mathrm{ml}$.

The pressor response to synthetic $\alpha$-L-asparaginy $1^{1}-$ valyl $^{5}$ angiotensin II (angiotensin amide) and to norepinephrine were compared in each of the 34 rats studied. In 30 of these rats, the pressor response to $\alpha$-L-asparty ${ }^{1}$-valyl ${ }^{5}$ angiotensin II (angiotensin acid) was also compared with that to norepinephrine. The dose of norepinephrine that would give a pressor response of $16 \mathrm{~mm} \mathrm{Hg}$ or less (the comparatively linear range of the dose response curve) was determined in each rat. After the blood pressure had returned to base line levels, the dose of angiotensin (amide or acid) that would give an identical rise in arterial pressure was found. Repeated responses were determined with each drug to assure reproductibility of the results. From these measurements the dosage ratio norepinephrine/angiotensin giving equipressor responses was calculated as an index of the relative responsivity to these agents. The dosage range for norepinephrine was $10-20 \mathrm{~m} \mu \mathrm{g}$, for angiotensin amide $2-4 \mathrm{~m} \mu \mathrm{g}$, and for angiotensin acid 0.8-10.5 $\mathrm{m} \mu \mathrm{g}$. The maximum fluid volume delivered was $0.04 \mathrm{ml}$.

26 of the rats were sacrificed after this procedure. After ligating the right carotid artery and the left jugular vein, the remaining 10 rats were saved for the studies described in Part II

Part II. Four groups of rats indentical with those in Part I were studied. Ten normal rats, six DOCA rats, eight $\mathrm{GN}$ rats, and fourteen $\mathrm{G}$ rats received immunization injections with angiotensin chemically linked to a carrier protein. Two DOCA rats, three $G N$ rats, and two $G$ rats received mock immunization injections. The antigen was prepared according to the method of Goodfriend, Levine, and Fasman (20) as modified by Oken and Biber (24) (Fig. 1). To $5 \mathrm{mg}$ of rat albumin (Pentex, Inc., Kankakee, Ill.) in $0.25 \mathrm{ml}$ of distilled water were dissolved $10 \mathrm{mg}$ of synthetic asparginyl valine $\mathbf{5}^{5}$ angiotensin II (Ciba Pharmaceutical Co., Summit, N. J.). $75 \mathrm{mg}$ of 1-cyclohexyl-3 (2 morpholinoethyl) carbodiimide (Aldrich Chemical Co., Inc., Milwaukee, Wis.) were dissolved in $0.125 \mathrm{ml}$ of distilled water. After mixing the two solutions and allowing them to stand at room temperature for $40 \mathrm{~min}$ to permit conjugation of the reactants to albumin, the mixture was dialyzed against 800 $\mathrm{ml}$ distilled water at $5^{\circ} \mathrm{C}$ for $14 \mathrm{hr}$. This antigen was then mixed thoroughly with an equal volume of Freund's adjuvant. The injectant for the mock immunized rats was prepared identically except that no angiotensin was added.

Each rat was injected three to nine times with $0.03 \mathrm{ml}$ of the mixture into the footpad at intervals of 7 days or more. In 16 rats a series of three or four immunizations were followed by a period of $30-100$ days during which time no injections were given. Reimmunization was then undertaken 
to study possible booster responses. Blood pressures (BP) were followed throughout the period of study. Each individual recorded $B P$ represents the average value of four readings obtained with duplicate recordings from each of two occluding tail cuffs of different size.

Rats were followed for up to 269 days after the onset of a stable hypertension with mean values of $170-230 \mathrm{~mm} \mathrm{Hg}$ recorded for at least 8 days before any immunization. During this control period, blood pressures were recorded three to seven times (average $4 \frac{1}{2}$ ). From 10 to 27 days after the final immunization series, the rats were prepared for direct $\mathrm{BP}$ recording as described in Part $\mathrm{I}$ and the pressor responses to angiotensin amide and to angiotensin acid were compared with that to norepinephrine. The results were expressed as norepinephrine/angiotensin equipressor dose ratios. In 10 rats, this pressor response was studied both before and after the period of immunization or mock immunization.

The plasmas from 20 rats were assayed for antibody against angiotensin according to the radioimmune assay method of Vallotton, Page, and Haber (25) by Dr. Lot B. Page. ${ }^{125} \mathrm{I}$ angiotensin purified with rabbit antiserum raised against poly-L-lysine-angiotensin copolymers was used. 50 $\mu 1$ of rat serum was incubated with approximately $0.02 \mathrm{~m} \mu \mathrm{g}$ ${ }^{125} \mathrm{I}$ angiotensin standard for $18 \mathrm{hr}$ at $4^{\circ} \mathrm{C}$. Bound angiotensin was precipitated from free angiotensin by ammonium sulfate. The results are expressed as bound to free $(B / F)$ ratios of angiotensin. A B/F ratio of greater than 0.2 is considered to represent the presence of antibody against angiotensin whereas a $\mathrm{B} / \mathrm{F}$ ratio of less than 0.2 represents nonspecific protein binding. Serial antibody determinations were not performed because of the mortality risk involved in this long-term study.

At the termination of the study, the kidneys of 23 of the renal hypertensive rats were assayed for renin according to the method of Boucher, Menard, and Genest (26). The individual kidneys were stripped of their capsule, weighed, homogenized, and the $\mathrm{pH}$ of the homogenate adjusted to 5.5 . After filtration of the homogenate, the filtrate was diluted $1: 200$ with normal saline and neomycin. One-tenth and 0.2 $\mathrm{ml}$ of the diluted filtrate was incubated at $37^{\circ} \mathrm{C}$ for $1 \mathrm{hr}$ in the presence of Dowex resin with $1 \mathrm{ml}$ of plasma ( $\mathrm{pH}$ adjusted to 5.5) from rats nephrectomized $24 \mathrm{hr}$ previously. Elution and bioassay was completed according to the method of Boucher et al. as modified by Blaufox, Birbari, Hickler, and Merrill (27). Results are expressed as millimicrograms of angiotensin per gram of kidney per min of incubation.

Statistical analysis. Student's $t$-test was used in the statistical evaluation of the data. Means are shown with \pm 1 SD.

\section{RESULTS}

Part I. Response to angiotensin in experimental hypertension

The dosage of norepinephrine required to give a blood pressure rise of $9-16 \mathrm{~mm} \mathrm{Hg}$ varied between rats. To obviate this the pressor variation ratios of norepinephrine/angiotensin amide, and of norepinephrine/ angiotensin acid required to give an equal blood pressure rise were determined. A high ratio results from increased sensitivity to angiotensin and a low ratio results from increased resistance to angiotensin. The group mean intrinsic vascular reactivity of the three groups of hypertensive rats under anesthesia was equivalent as measured by the amount of norepinephrine required to give the same blood pressure rise (Table I). Therefore, the difference in the response to angiotensin cannot be attributed to varying states of cardiovascular reactivity.

Table I compares the norepinephrine/angiotensin ratios for each group of rats. In normal and GN rats the mean norepinephrine/angiotensin amide ratios were similar whereas the DOCA rats had a significantly higher mean ratio $(P<0.001)$, which showed increased sensitivity to angiotensin amide; the $\mathrm{G}$ rats had a significantly lower ratio $(P<0.001)$, showing marked resistance to angiotensin amide.

Although the group differences with angiotensin acid are similar to those seen with angiotensin amide, there is a greater spread of the individual points. The GN rats were more resistant to angiotensin acid than were normal rats $(P=0.005)$ and less resistant than were $\mathrm{G}$ rats $(P=0.05)$. On comparison with normal rats, the DOCA rats did not have a significantly greater sensitivity to angiotensin acid whereas the $\mathrm{G}$ rats were highly resistant $(P<0.001)$. DOCA rats were more sensitive than either $\mathrm{GN}$ rats $(P<0.005)$ or $\mathrm{G}$ rats $(P<$ 0.001 ).

Part II. Immunization of rats with a conjugate of angiotensin

A fall in blood pressure of $30 \mathrm{~mm} \mathrm{Hg}$ or more $(>2$ sD of our observed spontaneous mean pressure variation in renovascular hypertensive rats) from average control levels and lasting for 8 or more days was considered significant and occurred after at least one series of immunizations in 8 of $14 \mathrm{G}$ rats $(57 \%)$ and three of eight GN rats (38\%). Four rats had significant booster responses after a subsequent series of immunizations, and three rats had blood pressures which fell significantly and never returned to control levels.

Significant blood pressure reduction occurred on the average of 42 days after the first injection in the first series of immunizations; in one rat the blood pressure fell significantly 38 days after a single immunization injection. Booster responses occurred on the average of 15 days after the first injection of the booster series. The average duration of a given blood pressure reduction was longer after the first series of immunizations (54 days) than after subsequent series (12 days).

Table II presents the following data: the total period each rat was followed; the average blood pressures before any immunization; the average blood pressures for the 10 days preceding termination of each study (time plasma taken for radioimmune assay); the change in blood pressure from the control period to the 10 days before completion of the study; the interval between the last series of immunization and death; and the presence 
of angiotensin antibody expressed as the bound/free ratios.

None of the immunized DOCA hypertensive rats and none of the mock immunized renovascular hypertensive rats had a significant blood pressure fall from control blood pressure levels during the period of observation. In fact, there was, in general, an increase in blood pressure during the period of observation in these groups,

TABLE I

Pressor Response of Normal Rats and Rats with Experimental Hypertension to Injections of Norepinephrine (NE), $\alpha$-L-asparaginyl-valine $e^{5}$ angiotensin $I I(A A)$, and $\alpha$-L-aspartyl-valine ${ }^{5}$ angiotensin $I I(A A c)$

\begin{tabular}{|c|c|c|c|c|c|c|c|c|c|}
\hline \multirow[b]{2}{*}{ Group* } & \multirow[b]{2}{*}{ Rat No. } & \multirow{2}{*}{$\begin{array}{c}\text { Ave BP } \\
\text { before } \\
\text { study }\end{array}$} & \multirow{2}{*}{$\begin{array}{l}\text { BP during } \\
\text { anesthesia }\end{array}$} & \multicolumn{3}{|c|}{$\begin{array}{l}\text { m } \mu \mathrm{g} \text { giving equal pressor } \\
\text { response }\end{array}$} & \multirow[b]{2}{*}{ BP rise } & \multicolumn{2}{|c|}{ Ratios } \\
\hline & & & & $\mathrm{NE}$ & $\mathbf{A A}$ & AAc & & NE/amide & NE/acid \\
\hline & & $m m \mathrm{Hg}$ & $m m H_{g}$ & & & & $m m \mathrm{Hg}$ & & \\
\hline \multirow[t]{9}{*}{ Normal } & 1 & - & 60 & 20 & 10.0 & 2.2 & 12.5 & 2.0 & 9.0 \\
\hline & 2 & - & 48 & 20 & 11.0 & 3.0 & 10.5 & 1.8 & 6.7 \\
\hline & 4 & - & 50 & 15 & 5.5 & 1.8 & 11 & 2.7 & 8.3 \\
\hline & 5 & - & 60 & 20 & 9.4 & 2.3 & 16 & 2.1 & 8.7 \\
\hline & 6 & - & 65 & 20 & 11 & 2.3 & 13 & 1.8 & 8.7 \\
\hline & 7 & - & 55 & 20 & 10 & 2.3 & 12 & 2.0 & 8.7 \\
\hline & 8 & - & 50 & 20 & 7.8 & 1.9 & 12.5 & 2.6 & 10.5 \\
\hline & 9 & 一 & 75 & 20 & 6.8 & - & 9.5 & 2.9 & - \\
\hline & Mean & & 57 & 19 & 8.9 & 2.3 & 12.1 & $2.2 \pm 04 \ddagger$ & $8.6 \pm 1.1 \ddagger$ \\
\hline \multirow[t]{8}{*}{ DOCA } & D1 & 215 & 80 & 10 & 3.4 & 1.5 & 15 & 3.0 & 6.7 \\
\hline & D3 & 220 & 40 & 20 & 7.1 & 1.8 & 9.5 & 2.8 & 11.1 \\
\hline & D4 & 215 & 50 & 20 & 5 & 2.9 & 14 & 4.0 & 7.0 \\
\hline & D6 & 185 & 80 & 10 & 2.1 & 0.8 & 15 & 4.8 & 12.5 \\
\hline & D7 & 200 & 90 & 10 & 2.0 & 0.9 & 10.5 & 5.0 & 11.1 \\
\hline & D12 & 200 & 60 & 10 & 2.9 & 0.9 & 9 & 3.5 & 11.1 \\
\hline & D14 & 210 & 65 & 10 & 2.6 & 0.9 & 12 & 3.8 & 11.1 \\
\hline & Mean & 206 & 66 & 12.9 & 3.6 & 1.4 & 12.1 & $3.8 \pm 0.9_{\ddagger}^{+}$ & $10.1 \pm 2.3 \ddagger$ \\
\hline \multirow[t]{8}{*}{ GN } & GN2 & 190 & 85 & 10 & 3.6 & 2.0 & 13 & 2.8 & 5.0 \\
\hline & GN3 & 180 & 65 & 20 & 11 & 2.7 & 11.5 & 1.8 & 7.4 \\
\hline & GN11 & 195 & 100 & 10 & 8.9 & 2.1 & 12 & 1.2 & 4.8 \\
\hline & GN13 & 190 & 85 & 20 & 7.5 & 2.5 & 12 & 2.7 & 8.0 \\
\hline & GN16 & 195 & 95 & 20 & 13.5 & 3.6 & 13.5 & 1.5 & 5.6 \\
\hline & GN18 & 190 & 90 & 10 & 4.3 & 1.5 & 11.5 & 2.3 & 6.7 \\
\hline & GN T & 200 & 70 & 20 & 10 & - & 13 & 2.0 & - \\
\hline & Mean & 191 & 84 & 15.7 & 8.4 & 2.4 & 12.3 & $2.0 \pm 0.6 \ddagger$ & $6.3 \pm 1.3 \ddagger$ \\
\hline \multirow[t]{13}{*}{ G } & G1 & 175 & 90 & 20 & 20 & - & 15 & 1.0 & - \\
\hline & G3 & 170 & 80 & 20 & 30 & 5 & 15 & 0.7 & 4 \\
\hline & G4 & 170 & 65 & 10 & 6.9 & 2.1 & 12 & 1.4 & 4.8 \\
\hline & G7 & 195 & 80 & 20 & 22 & - & 10 & 0.9 & - \\
\hline & G8 & 175 & 80 & 10 & 6.7 & 1.25 & 13 & 1.5 & 8.0 \\
\hline & G9 & 180 & 90 & 10 & 20 & 5 & 12 & 0.5 & 2.0 \\
\hline & G11 & 230 & 120 & 10 & 7.5 & 1.25 & 15 & 1.3 & 8.0 \\
\hline & G13 & 190 & 50 & 20 & 41 & 10.5 & 11.5 & 0.5 & 1.9 \\
\hline & G14 & 200 & 110 & 10 & 10 & 2 & 12 & 1.0 & 5.0 \\
\hline & G18 & 240 & 75 & 20 & 42 & 12.5 & 12.5 & 0.4 & 1.6 \\
\hline & G19 & 260 & 100 & 10 & 37.5 & 5.9 & 10 & 0.3 & 1.7 \\
\hline & G23 & 220 & 75 & 20 & 33 & 8.0 & 9 & 0.6 & 2.5 \\
\hline & Mean & 200 & 85 & 13.5 & 23 & 5.4 & 12.2 & $0.8 \pm 0.4 \ddagger$ & $4.0 \pm 2.5 \ddagger$ \\
\hline
\end{tabular}

* See text for description of hypertensive rats.

$\ddagger$ Mean \pm 1 SD. 


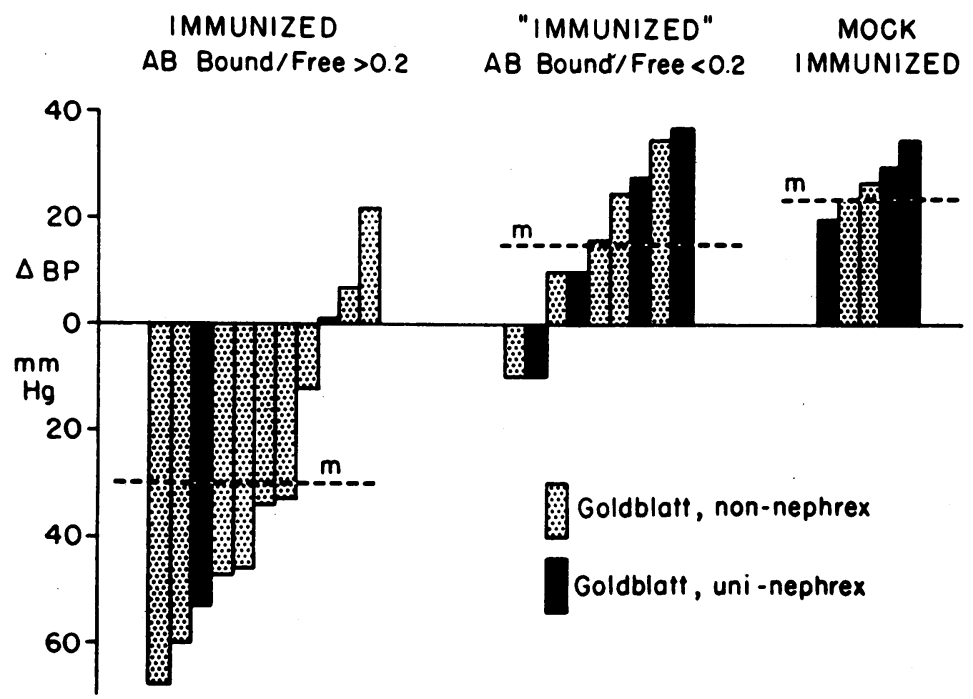

FIGURE 2 Change in blood pressure between control levels and levels recorded at time of antibody determination in Goldblatt rats. AB (antibody) bound/free $>0.2$ represents presence of antibodies whereas $A B$ (antibody) bound/free $<0.2$ represents nonspecific binding. Nonnephrex $=$ nonnephrectomized; uninephrex $=$ uninephrectomized.

indicating progression of the hypertensive process; there was an average increase of $21 \mathrm{~mm} \mathrm{Hg}$ in the immunized DOCA rats and $24 \mathrm{~mm} \mathrm{Hg}$ in the mock immunized $\mathrm{G}$ and $\mathrm{GN}$ rats. By contrast, of the 20 immunized renovascular hypertensive rats in whom antibodies were determined, 7 of the 11 in which a $B / F$ ratio $>0.2$ developed did show a significant reduction in blood pres- sure. This included one $\mathrm{GN}$ and six $\mathrm{G}$ rats. The remaining nine (four $\mathrm{GN}$ and five $\mathrm{G}$ ) had a $\mathrm{B} / \mathrm{F}$ ratio of $<0.2$, and none had a significant $\mathrm{BP}$ reduction. In fact, seven of the nine showed a BP increase, similar to the immunized DOCA rats and mock immunized renovascular hypertensive rats. Fig. 2 summarizes these changes. Additionally, of the four rats which did not show a sig-

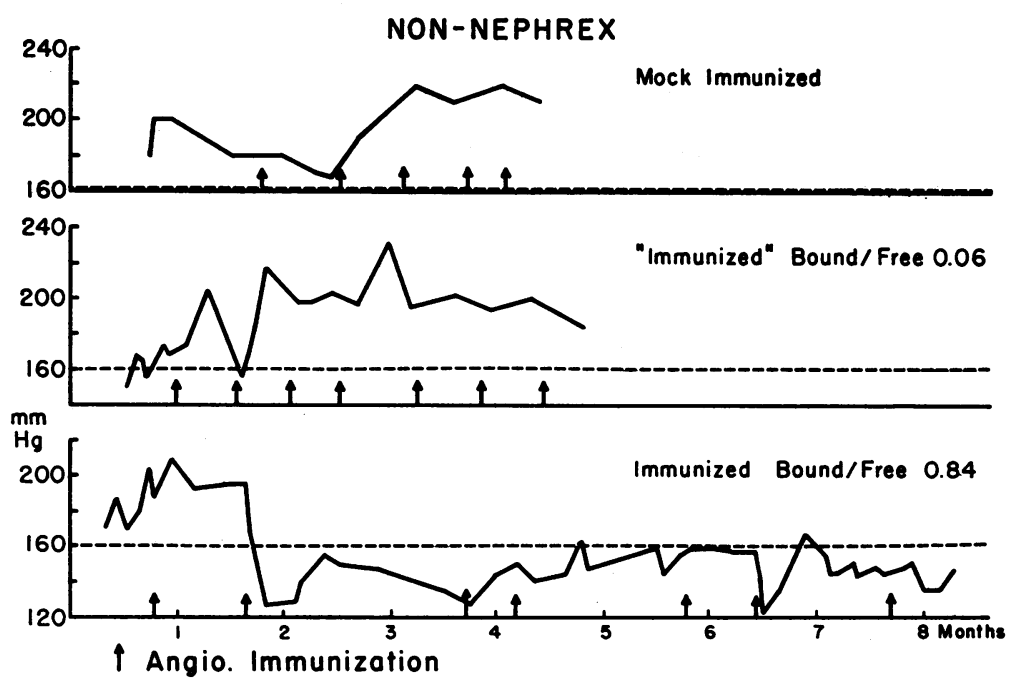

FiguRE 3 Blood pressure response and times of immunizations in each of three immunized or mock immunized rats rendered hypertensive by clipping one renal artery (G-rats). A bound/free ratio of $>0.2$ indicates the presence of angiotensin antibodies. A blood pressure fall of $30 \mathrm{~mm} \mathrm{Hg}$ or more and lasting for 8 days or more is considered significant. Nonnephrex $=$ nonnephrectomized. 
TABLE II

Relationship Between Antibody and Blood Pressure Change from Control Period to the 10 day Period before Antibody Determination

\begin{tabular}{|c|c|c|c|c|c|c|c|c|c|c|c|c|}
\hline & \multirow[b]{2}{*}{ Rat } & \multirow{2}{*}{$\begin{array}{l}\text { Duration } \\
\text { studied* }\end{array}$} & \multirow{2}{*}{$\begin{array}{l}\text { Total } \\
\text { inocu- } \\
\text { lations }\end{array}$} & \multirow{2}{*}{$\begin{array}{c}\text { Time } \\
\text { between } \\
\text { final } \\
\text { inocu- } \\
\text { lations } \\
+ \text { AB } \\
\text { determi- } \\
\text { nation } \\
\text { or death }\end{array}$} & \multicolumn{3}{|c|}{$\begin{array}{l}\text { Control BP before } \\
\text { inoculation }\end{array}$} & \multicolumn{3}{|c|}{$\begin{array}{l}\text { BP } 10 \text { days before } \\
\text { antibody determina- } \\
\text { tion or death }\end{array}$} & \multirow{2}{*}{$\begin{array}{c}\text { BP } \\
\text { change }\end{array}$} & \multirow{2}{*}{$\begin{array}{l}\text { Anti- } \\
\text { body } \\
\text { B/F } \\
\text { Ratioł }\end{array}$} \\
\hline & & & & & Mean & High & Low & Mean & High & Low & & \\
\hline & & days & & days & & $m m \mathrm{Hg}$ & & & $m m H g$ & & $m m H g$ & \\
\hline \multirow{13}{*}{$\begin{array}{c}\text { G + GN rats\& with } \\
\text { a } B / F \text { ratio } \neq>0.2\end{array}$} & & & & & & & & & & & & \\
\hline & G14 & 102 & 5 & 12 & 193 & 195 & 185 & 125 & 145 & 105 & -68 & 0.20 \\
\hline & G24 & 269 & 8 & 12 & 185 & 200 & 180 & 151 & 165 & 145 & -34 & 0.36 \\
\hline & G30 & 238 & 7 & 19 & 193 & 200 & 185 & 200 & 200 & 200 & +7 & 0.57 \\
\hline & G25 & 240 & 8 & 14 & 203 & 220 & 180 & 157 & 170 & 145 & -46 & 0.40 \\
\hline & G26. & 242 & 7 & 24 & 187 & 205 & 170 & 140 & 145 & 135 & -47 & 0.84 \\
\hline & G27 & 244 & 8 & 25 & 196 & 210 & 185 & 163 & 170 & 150 & -33 & 0.34 \\
\hline & G28 & 246 & 8 & 27 & 230 & 245 & 210 & 170 & 170 & 170 & -60 & 1.47 \\
\hline & G20 & 225 & 8 & 26 & 195 & 210 & 180 & 183 & 200 & 160 & -12 & 0.25 \\
\hline & G31 & 216 & 8 & 26 & 208 & 230 & 195 & 230 & 235 & 225 & +22 & 0.47 \\
\hline & GN25 & 203 & 7 & 14 & 193 & 205 & 180 & 140 & 145 & 130 & -53 & 0.27 \\
\hline & GN24 & 226 & 8 & 25 & 202 & 215 & 190 & 203 & 210 & 200 & +1 & 0.92 \\
\hline & Mean & $\overline{223}$ & $\overline{7}$ & $\overline{20}$ & $\overline{199}$ & $\overline{212}$ & $\overline{185}$ & $\overline{169}$ & $\overline{177}$ & $\overline{160}$ & $\overline{-30}$ & $\overline{0.55}$ \\
\hline \multirow{11}{*}{$\begin{array}{l}\text { G + GN rats§ with } \\
\text { a } \mathrm{B} / \mathrm{F} \text { ratio } \neq<0.2\end{array}$} & & & & & & & & & & & & \\
\hline & G11 & 104 & 6 & 13 & 230 & 240 & 225 & $240 \|$ & - & - & +10 & 0 \\
\hline & G1 & 128 & 7 & 13 & 172 & 175 & 170 & 188 & 190 & 185 & +16 & 0.06 \\
\hline & G7 & 124 & 6 & 10 & 195 & 210 & 180 & 220 & 225 & 215 & +25 & 0 \\
\hline & G8 & 190 & 10 & 22 & 178 & 180 & 175 & 213 & 235 & 200 & +35 & 0.08 \\
\hline & G33 & 152 & 6 & 26 & 210 & 220 & 200 & $200 \|$ & - & 一 & -10 & 0.13 \\
\hline & GN6 & 191 & 9 & 22 & 203 & 220 & 185 & 231 & 250 & 220 & +28 & 0.11 \\
\hline & GN 18 & 129 & 6 & 14 & 195 & 205 & 180 & 205 & 210 & 200 & +10 & $\mathbf{0}$ \\
\hline & GN22 & 236 & 8 & 25 & 183 & 215 & 170 & 173 & 185 & 145 & -10 & 0.06 \\
\hline & GN2 & 122 & 6 & 18 & 188 & 200 & 170 & 225 & 230 & 220 & +37 & 0 \\
\hline & Mean & 153 & 7 & 18 & 195 & 207 & 184 & 210 & 218 & 203 & +15 & 0.04 \\
\hline \multirow{4}{*}{$\begin{array}{l}\text { GN rats\& without } A B \\
\text { determination } \ddagger\end{array}$} & & & & & & & & & & & & \\
\hline & GN17 & 218 & 6 & 27 & 193 & 200 & 180 & 265 & 280 & 240 & +72 & - \\
\hline & GN13 & 106 & 6 & 17 & 188 & 200 & 175 & $225 \|$ & - & - & +37 & - \\
\hline & Mean & 162 & 6 & 22 & 191 & 200 & 178 & 245 & 253 & 233 & +55 & \\
\hline \multirow{7}{*}{$\begin{array}{l}\text { G + GN ratsई, mock } \\
\text { immunized }\end{array}$} & & & & & & & & & & & & \\
\hline & G13 & 117 & 5 & 16 & 188 & 200 & 175 & 212 & 220 & 205 & +24 & - \\
\hline & GN3 & 62 & 3 & 15 & 190 & 215 & 170 & 225 & 230 & 220 & +35 & - \\
\hline & GN16 & 135 & 6 & 20 & 218 & 220 & 180 & 238 & 250 & 225 & +20 & - \\
\hline & GN11 & 138 & 6 & 25 & 202 & 215 & 190 & 232 & 245 & 220 & +30 & - \\
\hline & G4 & 131 & 5 & 30 & 170 & 180 & 160 & 197 & 220 & 185 & +27 & - \\
\hline & Mean & 117 & 5 & 21 & 193 & 206 & 175 & 217 & 233 & 211 & +27 & \\
\hline \multirow{9}{*}{$\begin{array}{l}\text { DOCA rats } \$ \text { without } \\
\text { AB determination } \ddagger\end{array}$} & & & & & & & & & & & & \\
\hline & D12 & 78 & 4 & 19 & 210 & 220 & 200 & 201 & 220 & 185 & -9 & - \\
\hline & D14 & 82 & 3 & 11 & 220 & 225 & 215 & 220 & 225 & 215 & 0 & - \\
\hline & D6 & 102 & 5 & 18 & 188 & 195 & 180 & $230 \|$ & - & 一 & +42 & - \\
\hline & D4 & 107 & 5 & 3 & 180 & 190 & 170 & 223 & 225 & 210 & +43 & - \\
\hline & D5 & 56 & 3 & 12 & 183 & 185 & 180 & 230 & 230 & 230 & +47 & - \\
\hline & D15 & 39 & 3 & 3 & 220 & - & - & 220 & 220 & 220 & 0 & - \\
\hline & & - & - & - & - & - & - & - & - & $\longrightarrow$ & $\longrightarrow$ & - \\
\hline & Mean & 77 & 4 & 11 & 200 & 206 & 194 & 221 & 225 & 215 & +21 & \\
\hline \multirow{4}{*}{$\begin{array}{l}\text { DOCA rats§, mock } \\
\text { immunized }\end{array}$} & & & & & & & & & & & & \\
\hline & D7 & 94 & 5 & 14 & 187 & 200 & 160 & 225 & 230 & 220 & +38 & - \\
\hline & D1 & 54 & 3 & 6 & 220 & 225 & 215 & $230 \|$ & - & - & +10 & - \\
\hline & Mean & $\overline{74}$ & - & $\overline{10}$ & $\overline{204}$ & $\overline{213}$ & $\overline{188}$ & $\overline{228}$ & $\overline{230}$ & $\overline{225}$ & $\overline{+24}$ & - \\
\hline
\end{tabular}

* Total study period after onset of hypertension.

$\ddagger \mathrm{AB}$ determination $=$ antibody expressed as $\mathrm{B} / \mathrm{F}$ (bound/free) ratio.

See text for description of hypertensive rats.

|| Solitary pressure determination during 10 days before termination of study. Previous pressures equal or higher. 
UNI-NEPHREX

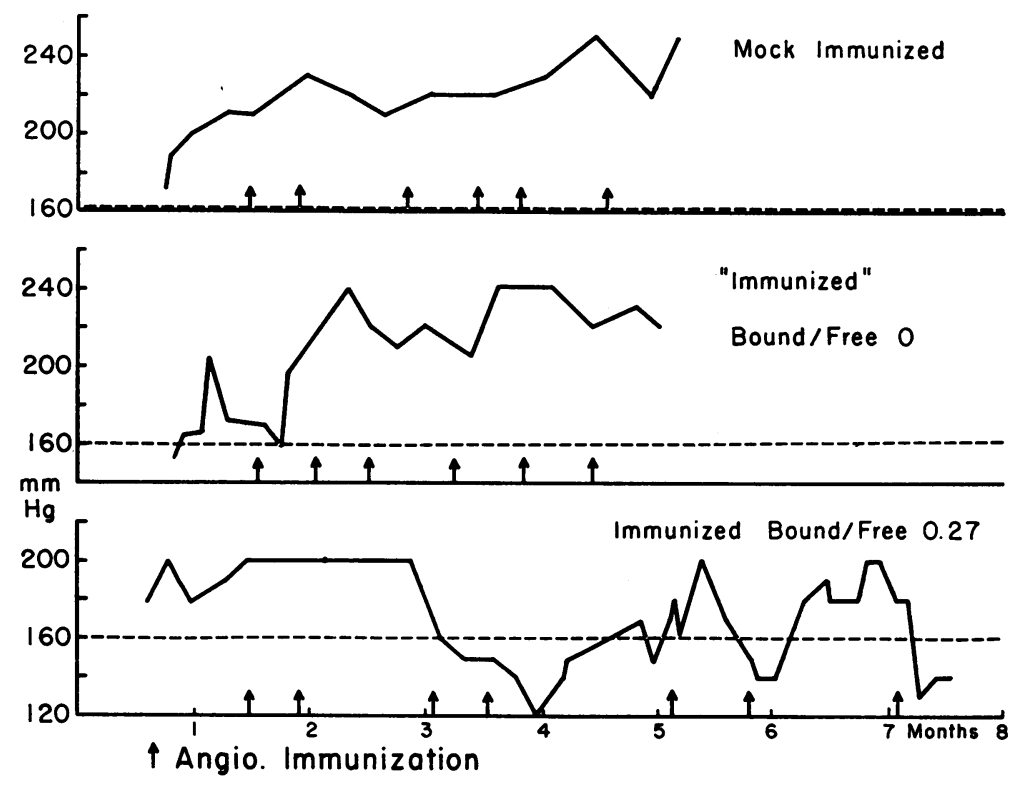

Figure 4 Blood pressure response and times of immunizations in each of three immunized or mock immunized rats rendered hypertensive by clipping one renal artery and contralateral nephrectomy (GN rats). A bound/free ratio of $>0.2$ indicates the presence of angiotensin antibodies. A blood pressure fall of $30 \mathrm{~mm} \mathrm{Hg}$ or more lasting for 8 days or more is considered significant. Uninephrex $=$ uninephrectomized.

nificant BP fall between control levels and the time of antibody determination despite a $\mathrm{B} / \mathrm{F}$ ratio of $>0.2$, 3 (Nos. G20, G31, and GN24, Table II) had shown a significant $\mathrm{BP}$ reduction earlier in the course. Of the nine rats with a $B / F$ ratio of $<0.2$, only one rat (No. GN22, Table II) had shown a significant blood pressure reduction earlier in its course. The immunized DOCA rats lost weight and died before pressor responses to angiotensin and norepinephrine were determined and without plasma radioimmune assay for antibodies. Rat Nos. GN17 and GN13 (Table II) also died without final studies.

Fig. 3 details the blood pressure response and the times of immunization or mock immunization in each of three representative $G$ rats. The course of rat No. G13, which was mock immunized and studied for 106 days, is seen in the upper plot. No significant blood pressure fall occurred. The course of rat No. G1, which received immunization injections but had no significant antibodies (B/F of 0.06 ) demonstrable at the end of the study, is shown in the center plot. This rat was followed for 128 days. Again, no significant blood pressure reductions occurred; as shown, the blood pressure actually increased during the period of observation. In the lower plot is seen the course of rat No. G26, which was followed for 187 days after which time a significant antibody $\mathrm{B} / \mathrm{F}$ ratio of 0.84 was found. A significant blood pressure fall occurred after the first series of immunizations. This rat retained a significantly lowered blood pressure throughout the period of study.

Similar results for three GN rats can be seen in Fig. 4. The upper plot shows the course of rat No. GN11, which was mock immunized and followed for 104 days. A persistent increase in blood pressure was observed over the period of observation. The center plot follows rat No. GN2 for a period of 188 days after which time no significant antibodies were measured $(B / F$ ratio of $0)$. There was no significant fall in blood pressure after any of the immunizations. The course of rat No. GN25, which was followed for 193 days is seen in the lower plot. A significant reduction in blood pressure occurred after the first immunization series. Further falls in BP ("booster responses") are seen after the second and third series of immunizations. At the end of the study at which time the blood pressure was $53 \mathrm{~mm} \mathrm{Hg}$ below control levels, a significant $\mathrm{B} / \mathrm{F}$ ratio of 0.27 was measured.

Pressor responsiveness of inoculated rats. After immunization of normal rats, the mean ratios fell from 2.2 to 1.0 with angiotensin amide $(P<0.001)$ (Tables I and III). These changes are consistent with those previously described (21) and reveal a highly significant increase in refractoriness to angiotensin amide as 
TABLE III

Pressor Response of Normal Rats and Rats with Experimental Hypertension to Inject ofions Norepinephrine (NE), $\alpha$-L-asparaginyl'-valine ${ }^{5}$ Angiotensin $I I(A A)$, and $\alpha$-L-aspartyl-valine $e^{5}$ Angiotensin $I I(A A C)$ after Inoculation with an Angiotensin Conjugate or Mock Immunization

\begin{tabular}{|c|c|c|c|c|c|c|c|c|c|c|}
\hline \multirow[b]{2}{*}{ Group* } & \multirow[b]{2}{*}{ Rat No. } & \multirow{2}{*}{$\begin{array}{l}\text { Total } \\
\text { inocu- } \\
\text { lations }\end{array}$} & \multirow{2}{*}{$\begin{array}{l}\text { Anti- } \\
\text { body } \\
\text { B/F } \\
\text { ratioł }\end{array}$} & \multirow{2}{*}{$\begin{array}{c}\text { BP } \\
\text { during } \\
\text { anesthe- } \\
\text { sia }\end{array}$} & \multicolumn{3}{|c|}{$\begin{array}{l}\text { m } \mu \mathrm{g} \text { giving equal pressor } \\
\text { response }\end{array}$} & \multirow[b]{2}{*}{$\mathrm{BP}$ rise } & \multicolumn{2}{|c|}{ Ratios } \\
\hline & & & & & NE & AA & AAc & & NE/AA & NE/AAc \\
\hline & & & & $m m \mathrm{Hg}$ & & & & $m m H g$ & & \\
\hline \multicolumn{11}{|c|}{ Inoculation with angiotensin conjugate } \\
\hline \multirow[t]{12}{*}{ Normal } & 1 & 4 & - & 65 & 20 & 7.5 & 一 & 12 & 2.7 & 一 \\
\hline & 2 & 7 & - & 40 & 20 & 27 & - & 8 & 0.7 & - \\
\hline & 3 & 6 & - & 115 & 30 & 49 & 21 & 9 & 0.6 & 1.4 \\
\hline & 5 & 7 & - & 60 & 20 & 50 & 15 & 11.5 & 0.4 & 1.3 \\
\hline & 6 & 4 & - & 50 & 20 & 60 & - & 12 & 0.3 & - \\
\hline & 7 & 4 & - & 75 & 10 & 7.5 & - & 12 & 1.3 & - \\
\hline & 8 & 5 & - & 70 & 20 & 20 & 5 & 10 & 1.0 & 4.0 \\
\hline & 10 & 6 & - & 70 & 20 & 11.8 & 11 & 10 & 1.7 & 1.8 \\
\hline & 11 & 7 & - & 50 & 40 & 51 & 69 & 8.5 & 0.8 & 0.6 \\
\hline & 12 & 6 & - & 60 & 20 & 30 & 23 & 10 & 0.7 & 0.9 \\
\hline & 50 & 6 & - & 75 & 20 & 24 & 13 & 11 & 0.8 & 1.5 \\
\hline & Mean & 6 & & 66 & 21.8 & 30.7 & 22.4 & 10.4 & $1.0 \pm 0.7 \S$ & $1.6 \pm 1.1 \S$ \\
\hline \multirow[t]{9}{*}{ GN } & GN25 & 7 & 0.27 & 50 & 40 & 46 & 22 & 10.5 & 0.9 & 1.8 \\
\hline & GN24 & 8 & 0.92 & 90 & 20 & 31 & 37.2 & 13 & 0.6 & 0.5 \\
\hline & GN6 & 9 & 0.11 & 90 & 20 & 15 & 3.9 & 8 & 1.3 & 5.1 \\
\hline & GN18 & 6 & 0 & - & - & - & - & - & - & - \\
\hline & GN22 & 8 & 0.06 & 85 & 20 & 8 & 3.8 & 11.5 & 2.5 & 5.3 \\
\hline & GN2 & 6 & 0 & 85 & 10 & 200 & 150 & 10 & 0 & 0.1 \\
\hline & GN17 & 6 & - & - & - & - & - & - & - & - \\
\hline & GN13 & 6 & - & - & - & - & - & - & - & - \\
\hline & Mean & 7 & & 80 & 22 & 60 & 43.4 & 10.6 & $1.1 \pm 09 . \S$ & $2.6 \pm 2.5 \S$ \\
\hline \multirow[t]{15}{*}{$\mathrm{G}$} & G14 & 5 & 0.20 & 55 & 20 & 64 & 14.5 & 16 & 0.3 & 1.4 \\
\hline & G24 & 8 & 0.36 & 60 & 20 & 18 & 7.3 & 8 & 1.1 & 2.7 \\
\hline & G30 & 7 & 0.57 & 85 & 20 & 15 & 6.4 & 9 & 1.3 & 3.1 \\
\hline & G25 & 8 & 0.40 & 50 & 20 & 100 & 49 & 8.5 & 0.2 & 0.4 \\
\hline & G26 & 7 & 0.84 & 50 & 30 & 49 & 15 & 9 & 0.6 & 2.0 \\
\hline & G27 & 8 & 0.34 & 50 & 20 & 10 & 5.9 & 11.5 & 2.0 & 3.4 \\
\hline & G28 & 8 & 1.47 & 90 & 20 & 20 & 7.4 & 11 & 1.0 & 2.7 \\
\hline & G20 & 8 & 0.25 & 80 & 20 & 7 & 3.8 & 9 & 2.8 & 5.3 \\
\hline & G31 & 8 & 0.47 & 75 & 20 & 31 & 12 & 8.5 & 0.7 & 1.7 \\
\hline & G11 & 6 & 0 & 120 & 10 & 20 & 6.8 & 10 & 0.5 & 1.5 \\
\hline & G1 & 7 & 0.06 & 70 & 20 & 20 & 5.5 & 8 & 1.0 & 3.6 \\
\hline & G7 & 6 & 0 & 100 & 10 & 16 & 2.9 & 8 & 0.6 & 3.5 \\
\hline & G8 & 10 & 0.08 & 70 & 20 & 17 & 3.4 & 12 & 1.2 & 4.5 \\
\hline & G33 & 6 & 0.13 & - & - & 一 & - & - & - & - \\
\hline & Mean & 7 & & 73 & 19.2 & 29.8 & 10.8 & 9.9 & $1.0 \pm 0.7 \S$ & $2.8 \pm 1.35 \S$ \\
\hline \multicolumn{11}{|c|}{ Mock immunized } \\
\hline \multirow[t]{3}{*}{ GN } & GN11 & 6 & - & 60 & 20 & 40 & 10 & 8 & 0.5 & 2.0 \\
\hline & GN16 & 6 & - & 100 & 20 & 13 & 3.6 & 11 & 1.5 & 5.6 \\
\hline & Mean & 6 & & 80 & 20 & 26.5 & 6.8 & 9.5 & 1.0 & 3.8 \\
\hline \multirow[t]{3}{*}{ G } & G13 & 5 & - & 65 & 10 & 28.5 & 5.3 & 8 & 0.4 & 1.9 \\
\hline & G4 & 5 & - & 80 & 10 & 8.3 & 1.9 & 9.5 & 1.2 & 5.2 \\
\hline & Mean & 5 & & 73 & 10 & 18.4 & 3.6 & 8.8 & 0.8 & 3.6 \\
\hline
\end{tabular}

* See text for description of hypertensive rats.

$\ddagger$ Antibody expressed as $\mathrm{B} / \mathrm{F}$ (bound/free) ratio where determined.

$\S$ Mean \pm 1 SD. 
a result of immunization. Previously (21) it was shown that no significant increase in angiotensin refractoriness occurred after mock immunization. Nonimmunized G rats had low mean ratios with angiotensin amide similar to the low mean ratios after "immunization" regardless of whether or not a B/F ratio of $>0.2$ was found. After immunization of GN rats, the mean ratios with angiotensin amide fell from 2.0 to $1.1(P<0.05)$ with no significant difference between those which developed antibodies and those which did not.

After immunization of normal rats, the mean ratios with angiotensin acid fell from 8.6 to $1.6(P<0.001)$. In $G$ rats the mean ratio fell from 4 to 2.8 (statistically not significant); the response was the same in those with and those without demonstrable antibodies. The nonimmunized GN rats had a mean ratio of 6.3 which fell to 2.6 after immunization $(P<0.01)$; the two rats with demonstrable antibodies had a mean ratio of 1.1 compared with a mean ratio of 3.7 in the three rats without demonstrable antibodies.

The pressor responsiveness as expressed by norepinephrine/angiotensin ratios was also studied both before and after immunization and mock immunization in each of 10 rats. Figs. 5 and 6 summarize these paired ratios for each rat. The mean norepinephrine/angiotensin amide ratio for all of the immunized rats so studied fell from $1.4 \pm 0.7$ to $0.6 \pm 0.5(P<0.025)$. For the $\mathrm{G}$ rats only, the mean ratio fell from $1.1 \pm 0.3$ to $0.7 \pm 0.4$ ( $P<$ 0.05 ). There was no significant difference between the mean norepinephrine/angiotensin amide ratios before and after mock immunization $(1.1 \pm 0.4$ and $0.9 \pm 0.5$ respectively). In three of these four rats, the ratio remained essentially unchanged after mock immunization. The mean norepinephrine/angiotensin acid ratio fell from $6.5 \pm 1.7$ to $1.9 \pm 1.9(P<0.01)$ in inoculated rats and was not dependent on the presence of demonstrable

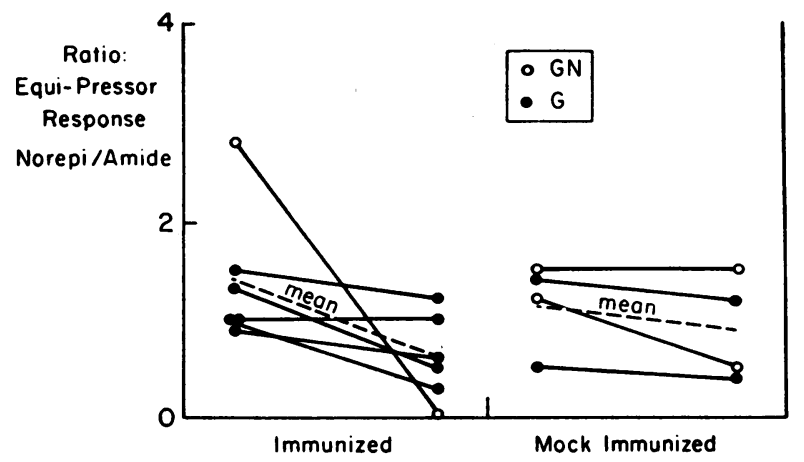

Figure 5 Pressor responsivity expressed as norepinephrine/angiotensin amide ratios before and after immunization or mock immunization in $\mathrm{G}$ and $\mathrm{GN}$ rats. A low ratio results from increased refractoriness to angiotensin.

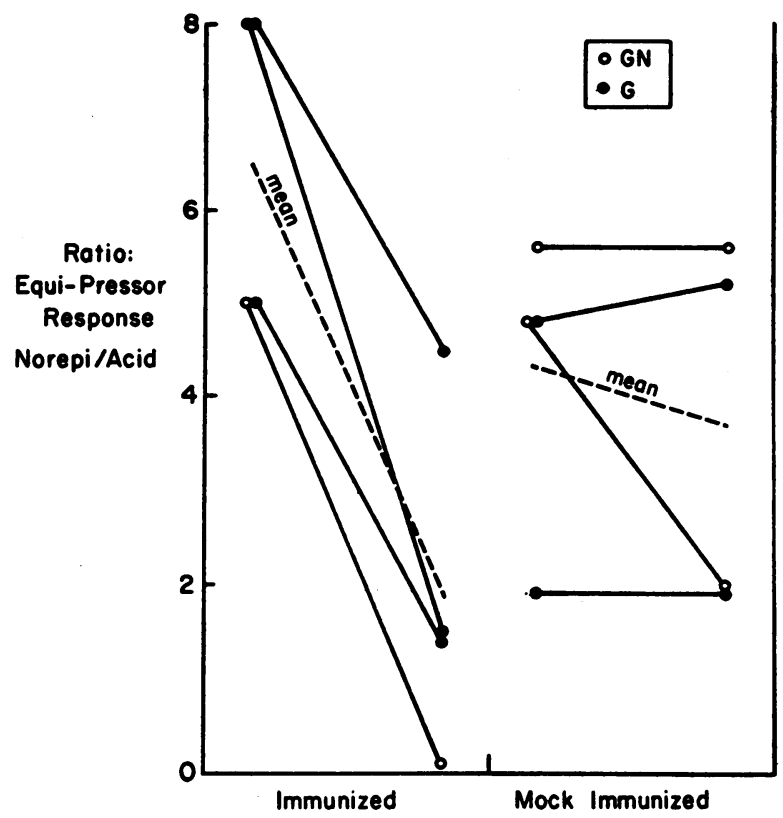

FIGURE 6 Pressor responsivity expressed as norepinephrine/ angiotensin acid ratios before and after immunization or mock immunization in $\mathrm{G}$ and $\mathrm{GN}$ rats. A low ratio results from increased refractoriness to angiotensin.

antibodies. With mock immunization, the norepinephrine/ angiotensin acid ratios did not change significantly (4.3 \pm 1.6 and $3.7 \pm 2.0$ respectively).

Renal renin activity. The results of the renal renin activity determined in the kidneys of immunized rats are shown in Table IV. In the G rats the renal renin activity in the clipped kidneys was consistently higher than in the unclipped kidney and was comparable in those with and those without demonstrable antibodies. By contrast, in rats with antibodies the mean renin activity in the unclipped kidneys was three times greater than that of the unclipped kidney in rats without antibodies. In the group with antibodies, the ratio of mean renal renin activity in the clipped to the unclipped kidney was 6 ; in the group without antibodies it was 15 . In the GN rats, the mean renal renin activity in those without antibodies was slightly higher than the mean renal renin activity of normal rats, and lower than that of the clipped kidney of $\mathrm{G}$ rats.

Plasma potassium. The plasma potassium and body weight of all rats with demonstrable antibodies and of six rats without demonstrable antibodies were determined. The group mean potassium was $3.2 \mathrm{mEq} /$ liter and $3.4 \mathrm{mEq} / \mathrm{liter}$ respectively (group mean of five normal rats was $3.2 \mathrm{mEq} /$ liter). Mean body weights were similar, $453 \mathrm{~g}$ and $503 \mathrm{~g}$ respectively. 
TABLE IV

Renal Renin Activity (RRA)* in Kidneys of Normal Rats and Renal Hypertensive Rats Inoculated with an Angiotensin Conjugate

\begin{tabular}{|c|c|c|c|c|c|c|c|c|c|c|c|}
\hline & & \multicolumn{6}{|c|}{ G rats $\ddagger$} & & & & \\
\hline & & & $\mathrm{B} / \mathrm{F}>0.2$ & & & $\mathrm{~B} / \mathrm{F}<0.2 \S$ & & & GN & & \\
\hline \multicolumn{2}{|c|}{ Normal rats } & \multirow[b]{2}{*}{ Rat No. } & \multirow{2}{*}{$\begin{array}{l}\text { Unclipped } \\
\text { kidney } \\
\text { RRA }\end{array}$} & \multirow{2}{*}{$\begin{array}{c}\text { Clipped } \\
\text { kidney } \\
\text { RRA }\end{array}$} & \multirow[b]{2}{*}{ Rat No. } & \multirow{2}{*}{$\begin{array}{l}\text { Unclipped } \\
\text { kidney } \\
\text { RRA }\end{array}$} & \multirow{2}{*}{$\begin{array}{l}\text { Clipped } \\
\text { kidney } \\
\text { RRA }\end{array}$} & \multicolumn{2}{|c|}{$\mathrm{B} / \mathrm{F}>0.28$} & \multicolumn{2}{|c|}{$B / F<0.28$} \\
\hline Rat No. & RRA & & & & & & & Rat No. & RRA & Rat No. & RRA \\
\hline 1 & 4150 & G14 & 1970 & - & G11 & 350 & 3850 & GN26 & 5311 & GN6 & 4395 \\
\hline 2 & 2790 & G24 & 270 & 2945 & G1 & 390 & - & & & GN18 & 8580 \\
\hline 3 & 3460 & G30 & 1139 & 15430 & G7 & 250 & 4600 & & & GN22 & 2635 \\
\hline 4 & 3855 & G25 & 565 & 4495 & G8 & 130 & 5035 & & & GN2 & 2010 \\
\hline 5 & 4270 & G26 & 385 & 3465 & G33 & 508 & 5720 & & & & \\
\hline 6 & 2525 & G27 & 2610 & 6030 & & & & & & & \\
\hline 7 & 2890 & G28 & 617 & 8990 & & & & & & & \\
\hline \multirow[t]{2}{*}{8} & 4730 & G20 & 442 & 6094 & & & & & & & \\
\hline & & G31 & 1582 & 5978 & & & & & & & \\
\hline Mean & 3583 & & 1064 & 6678 & - & 326 & 4801 & & 5311 & & 4405 \\
\hline
\end{tabular}

* RRA expressed as millimicrograms angiotensin per gram kidney per minute of incubation.

$\ddagger$ See text for description of hypertensive rats.

$\S$ Antibody expressed as $\mathrm{B} / \mathrm{F}$ (bound/free) ratio.

\section{DISCUSSION}

Pathophysiologic studies in renal hypertension. The three types of experimental hypertension studied were selected because each type is reported to have a different level of both renal and circulating renin activity. In rats made hypertensive with salt loading and deoxycorticosterone (DOCA rats), the renal and peripheral renin activities are low $(28,29)$. Mechanisms to account for this type of hypertension have been reviewed recently (30). In experimental renal hypertension produced by clipping one renal artery and contralateral nephrectomy (GN rats), the renal and circulating renin activities are normal $(28,29,31)$. In experimental renal hypertension produced by clipping one renal artery with the contralateral kidney intact ( $\mathrm{G}$ rats), there is elevated renin activity in the clipped kidney and in the circulation but depressed renin activity in the untouched kidney $(28,29,31)$. Recent studies have shown a good correlation between changes in plasma renin activity and the concentration of angiotensin in the blood (32, 33).

An inverse relationship has been noted between the magnitude of the blood pressure response to exogenous angiotensin and renin activity (28). The results in Part I of the current study are generally in accord with this. DOCA rats were highly sensitive to both angiotensin amide and acid presumably reflecting the suppression of the renin-angiotensin system in this form of hypertension. $\mathrm{G}$ rats were highly resistant to both peptides, presumably due to high levels of circulating angiotensin.
GN rats however, which are reported to have normal renal renin activity (31) had normal pressor responsiveness to injected angiotensin amide, but had significantly decreased pressor responsiveness to angiotensin acid. It is probable that the natural angiotensin in rats is aspartyl angiotensin, and these results suggest that in GN rats the circulating angiotensin level may be higher than in normal rats but lower than in G rats.

The results of Part II indicate that the hypertension in the two forms of experimental renal hypertension can be ameliorated after successful in vivo production of antibodies against angiotensin, strongly suggesting that angiotensin is directly involved in the mechanism of the hypertension.

In this study immunization was started during the 1st month of hypertension in most of the rats. However, booster inoculations resulted in blood pressure falls as long as 8 months after the onset of stable hypertension. It appears, therefore, that immunization against angiotensin can result in blood pressure reduction in both the acute and the chronic phase of experimental renal hypertension.

Injections of angiotensin coupled to albumin through carbodiimide cross-linkage reversed the hypertensive process in $50 \%$ of the rats with experimental renal hypertension. Further, there was excellent correlation between the presence of antibody, expressed as bound/free ratios using the radioimmune assay, and the difference between the average control blood pressures taken before any immunization injections and the average blood 
pressures recorded for 10 days preceding antibody determination. 7 of 11 rats $(64 \%)$ with a bound/free ratio of $>0.2$ had a significant blood pressure fall between these periods. Although four of the rats with a bound/ free ratio of $>0.2$ did not have a similar blood pressure fall between these periods, three of the four did not have the progressive increase in blood pressure seen in the rats with a bound/free ratio of $<0.2$ and in the mock immunized rats. In addition, three of these four rats had shown a significant blood pressure reduction earlier in their course. Booster injections with the antigen after the blood pressure had returned to control hypertensive levels resulted in repeated significant blood pressure falls in four rats.

It is conceivable that the blood pressure reduction observed in the rats with demonstrable antibodies was a nonspecific consequence of successful immunization. However, it is unlikely that the blood pressure reduction resulted from a nonspecific effect of the immunization procedure itself since none of the nine rats with experimental renal hypertension which had a bound/ free ratio of $<0.2$ and none of the five mock immunized rats with experimental renal hypertension had a significant fall in blood pressure between average control levels and the average levels for the 10 days preceding antibody determination. Most of these rats had a gradual increase in blood pressure over the period of study, consistent with the natural progression of renal hypertension. That none of the six rats with hypertension produced with saline and deoxycorticosterone acetate had a significant reduction in blood pressure after the attempt to actively immunize them against angiotensin lends further evidence for the specificity of angiotensin antibodies in effecting the blood pressure fall seen in the renal hypertensive rats. Although no antibody determinations were obtained in these rats because of their premature death, it is possible that none developed antibodies. This appears unlikely, however, because more than $50 \%$ of the Goldblatt rats had a bound/free antibody ratio of $>0.2$.

The results of these studies suggest that angiotensin probably is the specific mediator in the hypertension associated with renal artery stenosis. Although aldosterone has been thought to play only a permissive role in the etiology of renovascular hypertension, the possibility exists that the reduction in blood pressure seen after immunization occurred as a consequence of a suppression of aldosterone secretion secondary to a decrease in circulating angiotensin. The normal plasma potassium in the group with and without demonstrable antibodies, and the similar body weights between the two groups suggest that mineralocorticoid activity was present. In addition, several studies have suggested that aldosterone secretion is not abolished following the removal of an- giotensin from the circulation and that aldosterone is not the mediator of renovascular hypertension $(16,34$ 38).

Comparable levels of hypertension resulted from either clipping one renal artery (high renin renal hypertension) or clipping one renal artery coupled with contralateral nephrectomy (normal or intermediate renin hypertension). The quantitative difference in the sensitivity to injected angiotensin found between the two preparations suggests a difference in the circulating level of angiotensin. This may be explained by invoking the antihypertensive function which has been postulated for the contralateral kidney, requiring that the clipped kidney secrete more renin (angiotensin) to sustain a comparable level of hypertension when both kidneys are present. It would appear, then, that angiotensin may be intimately involved in the initiation and maintenance of both forms of experimental renal hypertension.

Immunologic considerations. The immunologic circumstances in this study are unique in that active immunization against an endogenously produced low molecular weight peptide, angiotensin, has resulted not only in the production of antibodies against angiotensin, but also in a physiological response (blood pressure reduction). Booster inoculations resulted in physiologic responses which were not typical of anamnestic antibody responses in that the delay before the blood pressure fall averaged 15 days and the blood pressure reduction was of short duration, averaging 12 days. In this immunologic situation where there is constant endogenous production of the hapten, antibody binding must keep pace with production to block the biological effects (blood pressure fall). Similarly, in vitro detection of antibody would be limited to some degree by the amount of endogenous angiotensin present and bound to the antibody.

Several studies involving haptens have demonstrated that exposure to the hapten preceding the active immunization may result in suppression of antibody production and that this immunologic unresponsiveness may be due to persistence of the hapten during the period of attempted immunization (39-42). Partial immunologic unresponsiveness might explain the failure to demonstrate antibodies in some of the inoculated rats and also explain the delay in blood pressure fall after booster immunization inoculations.

It is postulated, therefore, that to achieve a surplus of antibody would take several days during which time angiotensin would have to be formed in greater amounts to maintain the hypertension. Similarly, once the blood pressure has fallen, the stimulus for angiotensin release would be augmented, resulting in a further increase in angiotensin and saturation of the antibody. The excess 
angiotensin would thereby produce renewed hypertension in a short period of time.

Evidence supporting this is found in the results of the renal renin activity determinations (Table IV) and the angiotensin injection tests (Table III). An elevated renal renin activity would suggest increased renin secretion and elevated angiotensin levels. An analysis of the renal renin activity levels in the clipped kidneys of the $\mathrm{G}$ rats fails to indicate any convincing difference between the group which did as opposed to the group which did not develop a bound to free antibody ratio of $>0.2$. Because these clipped kidneys are constantly stimulated to produce renin, this similarity between the two groups may be expected. By contrast, in the unclipped kidneys of the $G$ rats, the average renal renin activity was three times as high in the group which did as compared with the group which did not develop a bound to free ratio of $>0.2$, suggesting an increased level of circulating angiotensin in the group with demonstrable antibodies. Such an increase in angiotensin may be sufficient to bind available angiotensin antibody binding sites in a short period of time and account for the short duration of the blood pressure falls after booster injections. This might also explain the modified blood pressure course seen in the rats with a $\mathrm{B} / \mathrm{F}$ ratio of $>0.2$ but which did not have a significant blood pressure fall at the time of antibody determination. Here it appears that although antibody binding sites sufficient to bind angiotensin were present earlier in the course when a blood pressure fall was recorded, at the time of antibody determination, angiotensin production was stimulated with a resultant antibody neutralization of only enough angiotensin to prevent progression of the hypertensive process.

With the exception of the rats with a clip on one renal artery ( $G$ rats), which were highly resistant to injected angiotensin before immunization, immunized rats developed significantly greater refractoriness to injected angiotensin amide and acid, whether or not antibodies were demonstrated, when compared with nonimmunized or mock immunized rats. When paired norepinephrine/angiotensin ratios were performed before and after immunization or mock immunization in the same rats (Figs. 5 and 6 ), a significantly increased refractoriness to both angiotensin amide and angiotensin acid was observed in the inoculated rats and was not dependent on the presence of demonstrable antibodies. No significant change in refractoriness was observed in the mock immunized rats studied similarly. There are two explanations which may account for these results. First, it is possible that a slow release of the angiotensin from the antigen complex may lead to angiotensin tachyphylaxis. The quantity of angiotensin in the injected antigen however was less than half that causing refrac- toriness to an acute angiotensin injection $1 \mathrm{hr}$ after discontinuation of a 12 day infusion in four of seven rats (43). In addition, there was no observed increase in blood pressure after inoculations suggesting that if angiotensin were released from the antigen complex, it was not released in pressor amounts. The second explanation is that the observed increase in refractoriness is consistent with antibody neutralization of angiotensin in all of the inoculated rats, including those in which no blood pressure reduction was observed. The rats without demonstrable antibodies by radioimmune techniques could well have had an excess of endogenous angiotensin at the time of in vitro antibody studies.

\section{ACKNOWLEDGMENTS}

We express our appreciation to Dr. Lot B. Page for performing the radioimmune assays for angiotensin, to Dr. Charles B. Carpenter for his assistance in the interpretation of the immunological aspects of this study, and to Dr. Benjamin J. Murawski for his assistance in the statistical analysis of the data.

This work was supported by U. S. Public Health Service Grant HE 11306-02 and by The John A. Hartford Foundation, Inc.

\section{REFERENCES}

1. Tigerstedt, R., and P. G. Bergman. 1898. Niere und Kreislauf. Scand. Arch. Physiol. 8: 223.

2. Goldblatt, H., J. Lynch, R. F. Hanzal, and W. W. Summerville. 1934. Studies on experimental hypertension. I. The production of persistent elevation of systolic blood pressure by means of renal ischemia. J. Exp. Med. 59: 347.

3. Harrison, T. R., A. Blalock, and M. F. Mason. 1936. Effects on blood pressure of injection of kidney extracts of dogs with renal hypertension. Proc. Soc. Exp. Biol. Med. 35: 38 .

4. Prinzmetal, M., and B. Friedman. 1936. Pressor effects of kidney extracts from patients and dogs with hypertension. Proc. Soc. Exp. Biol. Med. 35: 122.

5. Braun-Menendez, E., J. C. Fasciolo, L. F. Leloir, and J. M. Munoz. 1939. La substancia hipertensora de la sangre del rinon isquemiado. Rev. Soc. Argent. Biol. 15: 420 .

6. Page, I. H., and O. M. Helmer. 1940. A crystalline pressor substance (angiotonin) resulting from the reaction between renin and renin activator. J. Exp. Med. 71: 29.

7. Laragh, J. H., M. Angers, W. G. Kelly, and S. Lieberman. 1960. Hypotensive agents and pressor substances. The effect of epinephrine, norepinephrine, angiotensin II and others on the secretory rate of aldosterone in man. J. Amer. Med. Ass. 174: 234.

8. Wakerlin, G. E., R. B. Bird, B. B. Brennan, M. H. Frank, S. Kremen, I. Kuperman, and J. H. Skom. 1953. Treatment and prophylaxis of experimental renal hypertension with renin. J. Lab. Clin. Med. 41: 708.

9. Kremen, S. H., and G. E. Wakerlin. 1955. Renin and antirenin in treatment of long term experimental renal hypertension in the dog. Proc. Soc. Exp. Biol. Med. 90: 99.

10. Frank, M. H., L. Graham, and G. E. Wakerlin. 1956. Treatment and prophylaxis of experimental renal hyper- 
tension in monkeys with renins and antirenins. Fed. Proc. 15: 66.

11. Helmer, O. M. 1958. Studies on renin antibodies. Circulation. 17: 648.

12. Wakerlin, G. E. 1958. Antibodies to renin as proof of the pathogenesis of sustained renal hypertension. Circulation. 17: 653 .

13. Deodhar, S. D., E. Haas, and H. Goldblatt. 1964. Production of antirenin to homologous renin and its effect on experimental renal hypertension. J. Exp. Med. 119: 425.

14. Katz, J. I., J. H. Skom, and G. E. Wakerlin. 1957. Pathogenesis of spontaneous and pyelonephritic hypertension in the dog. Circ. Res. 5: 137.

15. Masson, G. M. C., C. Kashii, M. Matsunaga, and I. H. Page. 1964. Hypertensive vascular disease produced by homologous renin. Science. 145: 178.

16. Masson, G. M. C., C. Kashii, M. Matsunaga, and I. H. Page. 1966. Hypertensive vascular disease induced by heterologous renin. Circ. Res. 18: 219.

17. Sen, S., R. R. Smeby, and F. M. Bumpus. 1968. Antihypertensive effect of an isolated phospholipid. Amer. J. Physiol. $214: 337$.

18. Deodhar, S. D. 1960. Immunologic production of antiangiotensin. I. Preparation of angiotensin-protein complex antigen. J. Exp. Med. 111: 419.

19. Deodhar, S. D. 1960. Immunologic production of antiangiotensin. II. Production and detection of antiangiotensin. J. Exp. Med. 111: 429.

20. Goodfriend, T. L., L. Levine, and G. D. Fasman. 1964. Antibodies to bradykinin and angiotensin: use of carbodiimides in immunology. Science. 144: 1344.

21. Haber, E., L. B. Page, and G. A. Jacoby. 1965. Synthesis of antigenic branch-chain copolymers of angiotensin and poly-L-lysine. Biochemistry. 4: 693.

22. Dietrich, F. M. 1966. Immunogenicity of synthetic angiotensin II. Int. Arch. Allergy Appl. Immunol. 30: 497.

23. Goodfriend, T., G. Fasman, D. Kemp, and L. Levine. 1966. Immunochemical studies of angiotensin. Immunochemistry. 3: 223.

24. Oken, D. E., and T. U. L. Biber. 1968. Biologically effective immunization against angiotensin. Amer. $J$. Physiol. 214: 791.

25. Vallotton, M. D., L. B. Page, and E. Haber. 1967. Radioimmunoassay of angiotensin in human plasma. Nature (London). 215: 714.

26. Boucher, R., J. Menard, and J. Genest. 1967. A micromethod for measurement of renin in the plasma and kidney of rats. Can. J. Physiol. Pharmacol. 45: 881.

27. Blaufox, M. D., A. E. Birbari, R. B. Hickler, and J. P. Merrill. 1966. Peripheral plasma renin activity in renalhomotransplant recipients. $N$. Engl. J. Med. 275: 1165.

28. Gross, F., H. Brunner, and M. Ziegler. 1965. Renin- angiotensin system, aldosterone, and sodium balance. Recent Progr. Hormone Res. 21: 119.

29. Gross, F., G. Schaechtelin, H. Brunner, and G. Peters. 1964. The role of the renin-angiotensin system in blood pressure regulation and kidney function. Can. Med. Ass. J. 90: 258 .

30. Hickler, R. B., E. A. Amsterdam, and A. R. Christlieb. 1968. Hypertension secondary to disorders of the adrenal cortex. In Cardiovascular Disorders. J. H. Moyer and A. N. Brest, editors. F. A. Davis Co., Philadelphia. 965.

31. Regoli, D., H. Brunner, G. Peters, and F. Gross. 1962. Changes in renin content in kidneys of renal hypertensive rats. Proc. Soc. Exp. Biol. Med. 109: 142.

32. Brown, J. J., A. F. Lever, J. I. S. Robertson, R. L. Hodge, R. D. Lowe, and J. R. Vane. 1967. Concurrent measurement of renin and angiotensin in the circulation of the dog. Nature (London). 215: 853.

33. Gocke, D. J., I. Oppenhoff, J. N. Gerten, L. M. Sherwood, and J. H. Laragh. 1968. Measurement of plasma angiotensin II and correlation with renin and aldosterone in normal and hypertensive man. J. Clin. Invest. 47: 40a.

34. Eilers, E. A., and R. E. Peterson. 1964. Aldosterone secretion in the rat. In Symposium on Aldosterone, Prague, 1963. E. E. Baulieu and P. Robel, editors. F. A. Davis Co., Philadelphia. 251.

35. Gross, F. 1960. Adrenocortical function and renal pressor mechanisms in experimental hypertension. In Essential Hypertension, An International Symposium. K. D. Bock and P. T. Cottier, editors. Springer-Verlag, Berlin. 33.

36. Cade, R., and T. Perenich. 1965. Secretion of aldosterone by rats. Amer. J. Physiol. 208: 1026.

37. Marieb, N. J., and P. J. Mulrow. 1964. Regulation of aldosterone secretion in the rat. Clin. Res. 12: 459.

38. Floyer, M. A. 1951. The effect of nephrectomy and adrenalectomy upon the blood pressure in hypertensive and normotensive rats. Clin. Sci. 10: 405.

39. Chase, M. W. 1946. Inhibition of experimental drug allergy by prior feeding of the sensitizing agent. Proc. Soc. Exp. Biol. Med. 61: 257.

40. Battisto, J. R., and J. Miller. 1962. Immunologic unresponsiveness produced in adult guinea pigs by parenteral introduction of minute quantities of hapten or protein antigen. Proc. Soc. Exp. Biol. Med. 111: 111.

41. Battisto, J. R., and M. W. Chase. 1963. Immunologic unresponsiveness to sensitization with simple chemical compounds. J. Exp. Med. 118: 1021 .

42. Battisto, J. R., and B. R. Bloom. 1966. Mechanism of immunologic unresponsiveness: a new approach. Fed. Proc. 25: 152.

43. Marieb, N. J., and P. J. Mulrow. 1965. The role of the renin-angiotensin system in the regulation of aldosterone secretion in the rat. Endocrinology. 76: 657. 